\title{
A single-blind, parallel trial of L-hydroxyproline in healthy adult subjects
}

\author{
Saori Akiduki ${ }^{1} \cdot$ Haruo Ito $^{2} \cdot$ Koji Morishita $^{3}$ Ayako Kamimura ${ }^{1}$
}

Received: 19 November 2014 / Accepted: 18 March 2015 / Published online: 1 April 2015

(C) The Author(s) 2015. This article is published with open access at Springerlink.com

As calcium oxalate is the most common constituent of urinary stones animal model of the urolithiasis has been established by feeding L-hydroxyproline, an endogenous precursor of oxalate. However, the influence of oral L-hydroxyproline on human urinary oxalate excretion has not been clearly demonstrated. We herein report a clinical study examining the influence of daily ingestion of L-hydroxyproline on urinary oxalate levels with a focus on measuring oxalic acid excreted in the urine.

In detail, we performed a single-blind, parallel designed study at Sta. Rosa Hospital and Medical Center, Philippines. This study was approved by the hospital review board and was in compliance with the Declaration of Helsinki. Forty healthy subjects (20 males and 20 females, $33.4 \pm 9.4$ years of age, who has no history of kidney disease) were divided into 4 groups with 10 subjects in each group with same sex ratio. L-Hydroxyproline doses of 0 , 500, 1000 and $2000 \mathrm{mg}$ were taken daily after breakfast for 12 weeks. Prior to and during each scheduled examination, subjects were reminded to refrain from intake of oxalate-rich foods and to drink more than $1 \mathrm{~L}$ of water per day. Medical interviews, physical examinations, vital sign measurements, anthropometric measurements, blood examinations and 24-h urine sample collections were

Ayako Kamimura

ayako.kamimura@kyowa-kirin.co.jp

1 Healthcare Products Development Center, KYOWA HAKKO BIO CO., LTD., 2, Miyukigaoka, Tsukuba, Ibaraki 305-0841, Japan

2 Department of Urology, Graduate School of Medicine, Chiba University, 1-8-1, Inohana Chuo Ward, Chiba 260-8677, Japan

3 External Relations Department, KYOWA HAKKO BIO CO., LTD., 1-6-1, Otemachi, Chiyoda-ku, Tokyo 100-8185, Japan conducted every 4 weeks at the hospital. A male and two female subjects were withdrawn from this study because of pregnancy or urinary tract infection so the final number of subjects was 37. Informed consent was obtained from qualified individuals. The baseline characteristics of subjects of each group were not different statistically. No adverse events were observed throughout the trial period except for a few symptoms like itchiness or headache which were self-reported from subjects, but the relationships with the test material were denied by the principle investigator.

On the 24-h urine test, almost no markers changed, but there was a significant change in total L-hydroxyproline excretions in the $2000 \mathrm{mg}$ L-hydroxyproline group compared to control group at the point of 8 weeks after first intake. Moreover, oxalic acid levels also increased significantly in the $2000 \mathrm{mg}$ group at the point of 8 and 12 weeks after first intake. However, all values returned to basal values after 4 weeks from the last L-hydroxyproline intake (Table 1). Various dietary factors can change urine oxalate excretion; one is a high-protein diet. Many studies have addressed urinary oxalate excretion after high-protein intake, but conclusions are still uncertain. For example, Knight et al. [1] not only showed significant changes in urinary oxalate with 5 and $10 \mathrm{~g}$ of gelatine administered to healthy subjects, but also indicated that the high-protein diet did not influence total daily urinary oxalate excretion [2]. Collagen contains approximately $10 \%$ of L-hydroxyproline, and the daily turnover of collagen in humans is estimated to be $2-3 \mathrm{~g} /$ day so the metabolism of L-hydroxyproline will be $240-420 \mathrm{mg}$ [1]. In this study, the urinary oxalate level following 500 or $1000 \mathrm{mg} /$ day L-hydroxyproline ingestion did not change compared with control but $2000 \mathrm{mg} /$ day L-hydroxyproline ingestion increased the urinary oxalate excretion, but the values did not exceed 
Table 1 Urinalysis results of 24-h urine sample

\begin{tabular}{|c|c|c|c|c|}
\hline \multirow[t]{3}{*}{ Urinalysis of $24-\mathrm{h}$ urine } & \multicolumn{4}{|c|}{ Subjects ( $n=37: 19$ male, 18 female) } \\
\hline & \multicolumn{4}{|l|}{ L-Hydroxyproline } \\
\hline & $\begin{array}{l}0 \mathrm{mg} / \mathrm{day} \\
(n=10: 5 \mathrm{M}, 5 \mathrm{~F})\end{array}$ & $\begin{array}{l}500 \mathrm{mg} / \mathrm{day} \\
(n=9: 5 \mathrm{M}, 4 \mathrm{~F})\end{array}$ & $\begin{array}{l}1000 \mathrm{mg} / \text { day } \\
(n=10,5 \mathrm{M}, 5 \mathrm{M})\end{array}$ & $\begin{array}{l}2000 \mathrm{mg} / \mathrm{day} \\
(n=8: 4 \mathrm{M}, 4 \mathrm{~F})\end{array}$ \\
\hline \multicolumn{5}{|l|}{$\mathrm{Na}$ (g/day) } \\
\hline Pre-intake & $3.1 \pm 1$ & $2.8 \pm 1.2$ & $3.2 \pm 0.9$ & $3 \pm 0.8$ \\
\hline 4 weeks after first intake & $3 \pm 1$ & $3.5 \pm 1.2$ & $3.6 \pm 1.1$ & $4 \pm 0.6$ \\
\hline 8 weeks after first intake & $2.3 \pm 1$ & $1.9 \pm 0.8$ & $3 \pm 1$ & $2.7 \pm 0.7$ \\
\hline 12 weeks after first intake & $3.3 \pm 1$ & $3.6 \pm 1.2$ & $3.3 \pm 0.9$ & $3.9 \pm 1.3$ \\
\hline 4 weeks after final intake & $3.5 \pm 1.6$ & $3.1 \pm 0.7$ & $3.4 \pm 0.8$ & $3.3 \pm 1.2$ \\
\hline \multicolumn{5}{|l|}{ K (g/day) } \\
\hline Pre-intake & $0.67 \pm 0.27$ & $0.66 \pm 0.28$ & $0.82 \pm 0.25$ & $0.72 \pm 0.24$ \\
\hline 4 weeks after first intake & $0.67 \pm 0.2$ & $0.6 \pm 0.27$ & $0.7 \pm 0.22$ & $0.81 \pm 0.25$ \\
\hline 8 weeks after first intake & $0.56 \pm 0.19$ & $0.55 \pm 0.3$ & $0.56 \pm 0.22$ & $0.61 \pm 0.18$ \\
\hline 12 weeks after first intake & $0.68 \pm 0.29$ & $0.68 \pm 0.24$ & $0.61 \pm 0.24$ & $0.71 \pm 0.28$ \\
\hline 4 weeks after final intake & $0.7 \pm 0.3$ & $0.59 \pm 0.29$ & $0.67 \pm 0.19$ & $0.66 \pm 0.3$ \\
\hline \multicolumn{5}{|l|}{$\mathrm{Ca}(\mathrm{g} /$ day $)$} \\
\hline Pre-intake & $0.16 \pm 0.07$ & $0.13 \pm 0.06$ & $0.12 \pm 0.04$ & $0.11 \pm 0.04$ \\
\hline 4 weeks after first intake & $0.19 \pm 0.08$ & $0.16 \pm 0.04$ & $0.17 \pm 0.05$ & $0.17 \pm 0.05$ \\
\hline 8 weeks after first intake & $0.12 \pm 0.04$ & $0.1 \pm 0.03$ & $0.12 \pm 0.05$ & $0.12 \pm 0.04$ \\
\hline 12 weeks after first intake & $0.14 \pm 0.08$ & $0.15 \pm 0.08$ & $0.13 \pm 0.05$ & $0.14 \pm 0.07$ \\
\hline 4 weeks after final intake & $0.13 \pm 0.07$ & $0.12 \pm 0.09$ & $0.12 \pm 0.05$ & $0.13 \pm 0.04$ \\
\hline \multicolumn{5}{|l|}{ Mg (g/day) } \\
\hline Pre-intake & $0.04 \pm 0.02$ & $0.05 \pm 0.03$ & $0.06 \pm 0.02$ & $0.04 \pm 0.01$ \\
\hline 4 weeks after first intake & $0.05 \pm 0.02$ & $0.05 \pm 0.02$ & $0.07 \pm 0.02$ & $0.06 \pm 0.02$ \\
\hline 8 weeks after first intake & $0.04 \pm 0.01$ & $0.05 \pm 0.02$ & $0.05 \pm 0.01$ & $0.05 \pm 0.02$ \\
\hline 12 weeks after first intake & $0.05 \pm 0.02$ & $0.06 \pm 0.02$ & $0.06 \pm 0.01$ & $0.05 \pm 0.02$ \\
\hline 4 weeks after final intake & $0.04 \pm 0.01$ & $0.05 \pm 0.02$ & $0.06 \pm 0.01 *$ & $0.05 \pm 0.02$ \\
\hline \multicolumn{5}{|l|}{ Inorganic phosphorus (g/day) } \\
\hline Pre-intake & $0.47 \pm 0.21$ & $0.41 \pm 0.18$ & $0.52 \pm 0.12$ & $0.47 \pm 0.1$ \\
\hline 4 weeks after first intake & $0.48 \pm 0.18$ & $0.45 \pm 0.12$ & $0.54 \pm 0.08$ & $0.55 \pm 0.16$ \\
\hline 8 weeks after first intake & $0.57 \pm 0.2$ & $0.49 \pm 0.1$ & $0.6 \pm 0.22$ & $0.59 \pm 0.13$ \\
\hline 12 weeks after first intake & $0.55 \pm 0.27$ & $0.51 \pm 0.2$ & $0.54 \pm 0.13$ & $0.51 \pm 0.17$ \\
\hline 4 weeks after final intake & $0.48 \pm 0.14$ & $0.41 \pm 0.18$ & $0.51 \pm 0.16$ & $0.49 \pm 0.16$ \\
\hline \multicolumn{5}{|l|}{ Uric acid (g/day) } \\
\hline Pre-intake & $0.19 \pm 0.06$ & $0.15 \pm 0.06$ & $0.17 \pm 0.08$ & $0.13 \pm 0.04$ \\
\hline 4 weeks after first intake & $0.19 \pm 0.05$ & $0.17 \pm 0.07$ & $0.17 \pm 0.06$ & $0.15 \pm 0.04$ \\
\hline 8 weeks after first intake & $0.12 \pm 0.03$ & $0.09 \pm 0.03$ & $0.12 \pm 0.06$ & $0.11 \pm 0.04$ \\
\hline 12 weeks after first intake & $0.15 \pm 0.05$ & $0.13 \pm 0.03$ & $0.14 \pm 0.04$ & $0.14 \pm 0.06$ \\
\hline 4 weeks after final intake & $0.16 \pm 0.03$ & $0.11 \pm 0.03$ & $0.14 \pm 0.05$ & $0.12 \pm 0.05$ \\
\hline \multicolumn{5}{|l|}{ Urea nitrogen (g/day) } \\
\hline Pre-intake & $6.14 \pm 2.78$ & $5.9 \pm 2.45$ & $6.7 \pm 2.02$ & $5.67 \pm 1.6$ \\
\hline 4 weeks after first intake & $6.74 \pm 2.44$ & $6.29 \pm 1.63$ & $7.17 \pm 1.37$ & $7.42 \pm 2.08$ \\
\hline 8 weeks after first intake & $7.04 \pm 2.65$ & $6.53 \pm 1.68$ & $7.19 \pm 1.9$ & $7.71 \pm 1.23$ \\
\hline 12 weeks after first intake & $6.84 \pm 2.92$ & $6.78 \pm 1.34$ & $6.96 \pm 1.58$ & $6.87 \pm 2.06$ \\
\hline 4 weeks after final intake & $6.08 \pm 1.43$ & $6.15 \pm 1.71$ & $6.73 \pm 1.24$ & $6.05 \pm 2.34$ \\
\hline \multicolumn{5}{|l|}{ Creatinine (g/day) } \\
\hline Pre-intake & $0.94 \pm 0.42$ & $0.82 \pm 0.33$ & $1 \pm 0.26$ & $0.93 \pm 0.28$ \\
\hline 4 weeks after first intake & $1.05 \pm 0.37$ & $0.93 \pm 0.21$ & $1.14 \pm 0.27$ & $1.13 \pm 0.33$ \\
\hline
\end{tabular}


Table 1 continued

\begin{tabular}{|c|c|c|c|c|}
\hline \multirow[t]{3}{*}{ Urinalysis of 24-h urine } & \multicolumn{4}{|c|}{ Subjects ( $n=37: 19$ male, 18 female) } \\
\hline & \multicolumn{4}{|l|}{ L-Hydroxyproline } \\
\hline & $\begin{array}{l}0 \mathrm{mg} / \mathrm{day} \\
(n=10: 5 \mathrm{M}, 5 \mathrm{~F})\end{array}$ & $\begin{array}{l}500 \mathrm{mg} / \mathrm{day} \\
(n=9: 5 \mathrm{M}, 4 \mathrm{~F})\end{array}$ & $\begin{array}{l}1000 \mathrm{mg} / \mathrm{day} \\
(n=10,5 \mathrm{M}, 5 \mathrm{M})\end{array}$ & $\begin{array}{l}2000 \mathrm{mg} / \mathrm{day} \\
(n=8: 4 \mathrm{M}, 4 \mathrm{~F})\end{array}$ \\
\hline 8 weeks after first intake & $1.18 \pm 0.46$ & $1.1 \pm 0.22$ & $1.28 \pm 0.32$ & $1.3 \pm 0.24$ \\
\hline 12 weeks after first intake & $1.05 \pm 0.47$ & $1.17 \pm 0.28$ & $1.15 \pm 0.26$ & $1.1 \pm 0.44$ \\
\hline 4 weeks after final intake & $1.11 \pm 0.34$ & $1.05 \pm 0.39$ & $1.18 \pm 0.34$ & $1.14 \pm 0.45$ \\
\hline \multicolumn{5}{|l|}{ Albumin (mg/g Cr) } \\
\hline Pre-intake & $2.1 \pm 0.4$ & $2.6 \pm 1.7$ & $1.9 \pm 0.5$ & $1.8 \pm 0.5$ \\
\hline 4 weeks after first intake & $2.1 \pm 1.1$ & $2 \pm 0.4$ & $1.7 \pm 0.5$ & $1.8 \pm 0.4$ \\
\hline 8 weeks after first intake & $1.9 \pm 0.9$ & $1.2 \pm 0.3$ & $1.7 \pm 0.9$ & $1.5 \pm 0.7$ \\
\hline 12 weeks after first intake & $1.9 \pm 0.9$ & $1.5 \pm 0.3$ & $3 \pm 4.5$ & $1.6 \pm 0.4$ \\
\hline 4 weeks after final intake & $3.5 \pm 4.9$ & $1.6 \pm 0.4$ & $1.5 \pm 0.5$ & $1.5 \pm 0.3$ \\
\hline \multicolumn{5}{|l|}{ Citric acid (mg/g Cr) } \\
\hline Pre-intake & $248.1 \pm 209.2$ & $244.4 \pm 156.5$ & $256.3 \pm 228.3$ & $212.7 \pm 181.2$ \\
\hline 4 weeks after first intake & $244.6 \pm 178.7$ & $203.8 \pm 137.4$ & $226.2 \pm 183.6$ & $175.1 \pm 132.4$ \\
\hline 8 weeks after first intake & $197.5 \pm 142.2$ & $180 \pm 150.7$ & $144.7 \pm 114.5$ & $134.9 \pm 118.8$ \\
\hline 12 weeks after first intake & $228.7 \pm 181.9$ & $189.2 \pm 142.2$ & $164.6 \pm 140.5$ & $155.5 \pm 146$ \\
\hline 4 weeks after final intake & $231.5 \pm 176.9$ & $190.6 \pm 120.4$ & $214.1 \pm 179.6$ & $165.5 \pm 118.3$ \\
\hline \multicolumn{5}{|l|}{ Hydroxyproline ( $\mu \mathrm{mol} /$ day $)$} \\
\hline Pre-intake & $210 \pm 89$ & $200 \pm 90$ & $232 \pm 65$ & $194 \pm 51$ \\
\hline 4 weeks after first intake & $218 \pm 68$ & $212 \pm 48$ & $256 \pm 48$ & $288 \pm 86$ \\
\hline 8 weeks after first intake & $253 \pm 96$ & $244 \pm 62$ & $288 \pm 72$ & $364 \pm 94 *$ \\
\hline 12 weeks after first intake & $207 \pm 103$ & $241 \pm 61$ & $263 \pm 93$ & $263 \pm 80$ \\
\hline 4 weeks after final intake & $236 \pm 88$ & $224 \pm 90$ & $246 \pm 52$ & $241 \pm 74$ \\
\hline \multicolumn{5}{|l|}{ Oxalic acid (mg/day) } \\
\hline Pre-intake & $15.1 \pm 7$ & $12 \pm 4.5$ & $15.6 \pm 4.1$ & $13.1 \pm 2.4$ \\
\hline 4 weeks after first intake & $24.8 \pm 7.8$ & $21.5 \pm 4.8$ & $27.2 \pm 5.6$ & $32.6 \pm 8.7$ \\
\hline 8 weeks after first intake & $17.3 \pm 4.6$ & $17 \pm 4.2$ & $21.5 \pm 6.8$ & $24.7 \pm 3.7^{*}$ \\
\hline 12 weeks after first intake & $19.2 \pm 7.7$ & $23.7 \pm 5.9$ & $25.2 \pm 5.1$ & $27.1 \pm 6.9^{*}$ \\
\hline 4 weeks after final intake & $18.1 \pm 6.4$ & $17.5 \pm 6.6$ & $18.7 \pm 4.7$ & $16.3 \pm 6.3$ \\
\hline
\end{tabular}

Mean group values and standard deviations were calculated and analyzed for homogeneity of variance using Bartlett's test (5\% level of significance). When the variance was homogeneous between the groups, mean group values were compared between the control and each treated group using Dunnett's test. When the variance was heterogeneous based on Bartlett's test, a mean rank test of the Dunnett type was conducted. Differences from the control group were evaluated at the two-tailed $5 \%$ level of significance and presented as $* p<0.05$

the level of stone forming individuals with a mean oxalate excretion $40 \mathrm{mg} / \mathrm{day}[2]$. As noted, there are various opinions on the association of dietary and urinary oxalate. Recently, it was reported that the Dietary-Approaches to Stop Hypertension (DASH)-style diet is more effective in reducing calcium oxalate supersaturation than a lowoxalate diet, although it includes a lot of fruits and vegetables which contain much oxalate [3]. Thus, the influence of the diet in conjunction with oxalate is still unclear in human metabolism. Moreover, we found no accumulation of L-hydroxyproline and oxalate in the withdrawal period same as the animal model. The metabolic significance of L-hydroxyproline is still unclear and further investigation is warranted.
Conflict of interest This study was funded by KYOWA HAKKO BIO CO., LTD. SA, KM and AK are employees of KYOWA HAKKO BIO CO., LTD. HI is a consultant of KYOWA HAKKO BIO CO., LTD.

Open Access This article is distributed under the terms of the Creative Commons Attribution License which permits any use, distribution, and reproduction in any medium, provided the original author(s) and the source are credited.

\section{References}

1. Knight J, Jiang J, Assimos DG, Holmes RP (2006) Hydroxyproline ingestion and urinary oxalate and glycolate excretion. Kidney Int 70(11):1929-1934 
2. Knight J, Easter LH, Neiberg R, Assimos DG, Holmes RP (2009) Increased protein intake on controlled oxalate diets does not increase urinary oxalate excretion. Urol Res 3(2):63-68

3. Noori N, Honarkar E, Goldfarb DS, Kalantar-Zadeh K, Taheri M, Shakhssalim N, Parvin M, Basiri A (2014) Urinary lithogenic risk profile in recurrent stone formers with hyperoxaluria: a randomized controlled trial comparing DASH (Dietary Approaches to Stop Hypertension)-style and low oxalate diets. Am J Kidney Dis 63(3):456-463 